\title{
Oral rehabilitation with implant-based prostheses of two adult patients treated for childhood rhabdomyosarcoma
}

\author{
Anke Korfage • Kees Stellingsma • Johan Jansma • \\ Arjan Vissink • Gerry M. Raghoebar \\ Received: 2 May 2011 / Accepted: 4 July 2011 /Published online: 13 July 2011 \\ (C) The Author(s) 2011. This article is published with open access at Springerlink.com
}

\begin{abstract}
Background Rhabdomyosarcoma is the most common malignant tumor in the nasal and paranasal sinus area at childhood. Multimodal treatment for this disorder has severe side effects due to normal tissue damage. As a result of this treatment, facial growth retardation and oral abnormalities such as malformation of teeth and microstomia can cause esthetic and functional problems.

Case reports Two cases are presented of patients with severe midfacial hypoplasia and reduced oral function as a result of treatment of rhabdomyosarcoma of the nasopharyngeal and nasal-tonsil region. With a combined surgical (osteotomy, distraction osteogenesis, implants) and prosthetic (implant-based overdenture) treatment, esthetics and function were improved.
\end{abstract}

Keywords Childhood rhabdomyosarcoma - Dental implants · Radiotherapy $\cdot$ Midfacial hypoplasia $\cdot$ Distraction osteogenesis

\section{Introduction}

Nasopharyngeal rhabdomyosarcoma is usually treated with a combination of chemotherapy, radiotherapy, and surgery [1]. Surgical resection of rhabdomyosarcoma is challenging and can result in large maxillofacial defects with loss of function and esthetics of the surrounding tissues. Radiotherapy may result in facial growth retardation, neuroendocrine dysfunc-

A. Korfage $(\bowtie) \cdot$ K. Stellingsma $\cdot$ J. Jansma $\cdot$ A. Vissink $\cdot$

G. M. Raghoebar

Department of Oral and Maxillofacial Surgery and Maxillofacial

Prosthetics, University Medical Center Groningen,

University of Groningen,

P.O. Box 30.001-BB70, 9700 RB Groningen, The Netherlands

e-mail: a.korfage@kchir.umcg.nl tion due to radiation injury of the pituitary gland, visual problems, and hearing loss [2, 3]. In addition, both chemotherapy and radiotherapy have widespread effects on oral tissues. These effects include delayed eruption of the teeth, root stunting, microdontia, hypodontia, discoloration, incomplete calcification of the teeth, microstomia, trismus, velopharyngeal insufficiency, and xerostomia [4-9].

As a result of the above-mentioned sequelae, it is not uncommon in childhood cancer survivors that teeth are lost at a later age [5]. Furthermore, the risk of developing dental caries among others can be increased due to reduced salivary flow, changes in the morphology of the enamel and dentine, and restricted possibilities for oral hygiene (microstomia, trismus). Loss of teeth in these patients will aggravate the already existing loss of oral function and poor esthetics. Moreover, with the loss of teeth, prosthetic treatment becomes even more challenging. Among others, the retention and stability of prostheses are likely to be impaired because the bone volume for good support and the tolerance of the denture-bearing mucosa to mechanical loading are reduced. Implant-based prosthodontics might resolve many of the limitations of conventional prosthodontics in a compromised oral situation [6].

In this paper, we present two cases of adult patients that were treated for rhabdomyosarcoma during childhood. Both patients had compromised oral function and esthetics due to severe side effects of their therapy. For both patients, a multidisciplinary treatment plan was made.

\section{Case reports}

Patient 1

A 24-year-old male was treated for a nasopharyngeal rhabdomyosarcoma at the age of four. Treatment consisted 
of surgical debulking of the tumor, followed by chemotherapy and interstitial radiotherapy (cumulative dose 44 Gy). This therapy resulted in a complete remission of the tumor. No recurrences occurred and neither did the patient develop metastases during a 20-year follow-up period. The late effects of the cancer treatment were delayed growth of the patient, hypoplasia of the midface (Fig. 1a), microdontia with root malformation (Fig. 1c), velopharyngeal insufficiency, and frequent ear infections with impaired hearing.

At the age of 12, a partial prosthesis (both dental and mucosal supported) with a velopharyngeal obturator was made to improve speech. The retention of this prosthesis was impaired due to the conical shape of the teeth and insufficient bone volume to support the prosthesis. The partial prosthesis had to be renewed after 4 years because of the growth of the maxillary complex resulting in a reduced fit of the prosthesis. Orthodontic therapy to correct the midfacial hypoplasia was contraindicated because of the already shortened roots of the teeth.

At the age of 18, i.e., after completion of facial growth, a combined surgical and prosthetic treatment plan was made to improve esthetics by correcting the midface hypoplasia and restoring the oral function. The treatment plan consisted of:

- Hyperbaric oxygen (HBO; 20 dives before and 10 dives after orthognatic surgery)

- Le Fort III osteotomy with placement of a rigid external distraction frame (RED) (KLS-Martin L.P., Jacksonville, FL, USA) with rigid plates secured to the infraorbital rim with transcutaneous wires to gradually distract the bones of the midface ventrally (Fig. 1a)

- Reconstruction of the hard and soft palate with a temporalis muscle flap

- Removal of the remaining upper teeth because of dental caries and mobility of the teeth due to the very short, underdeveloped roots

- Dental implant placement

- Prosthetic rehabilitation

The patient agreed with this treatment and the surgical procedure started at the age of 19 . Ten days after placement of the RED frame, the distraction started at a rate of $0.5 \mathrm{~mm}$ once daily (Fig. 1a). The vector of distraction osteogenesis (DO) was parallel to the Frankfort horizontal plane. Because of little progression, the rate of DO was increased to $0.5 \mathrm{~mm}$ twice a day from day 11 . After 25 days, active DO was stopped because a satisfying esthetic result was achieved according to the patient and the surgeon (Fig. 1b). The total advancement measured on lateral radiographs at the central incisors was $15 \mathrm{~mm}$.

After a 3-month consolidation period, the frame was removed. As a result of the forward movement of the maxilla, speech had deteriorated due to worsening of the velophar-
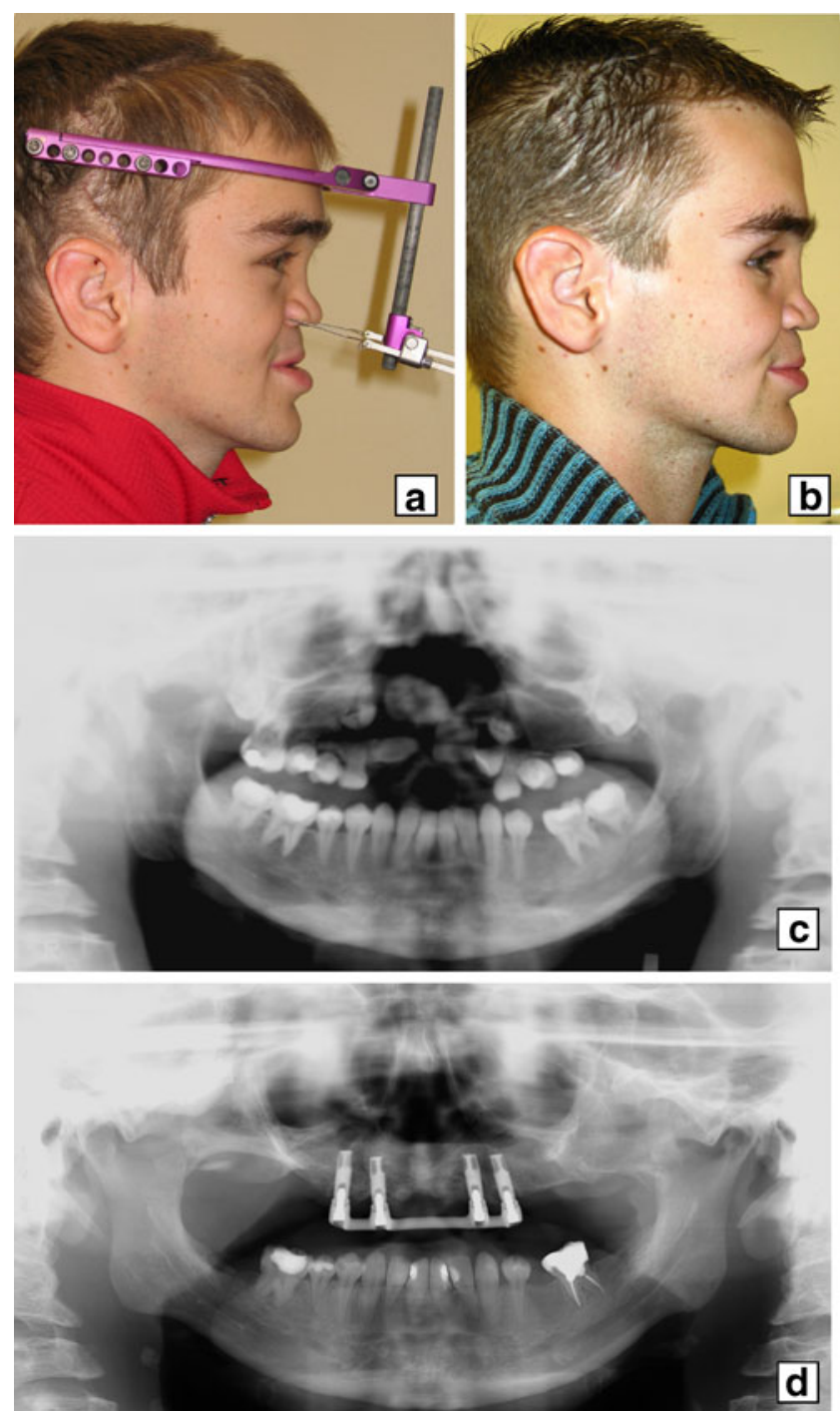

Fig. 1 a Patient at the age of 19 with hypoplasia of the midface due to delayed growth. A rigid external distraction frame (RED) was placed to correct this hypoplasia. b Patient at the age of 23 after correction of the midfacial hypoplasia and completion of prosthetic treatment showing a more ventral position of the midface and improved esthetics. c Rotational panoramic radiograph of the patient at the age of 13 showing hypodontia, conical shape of the crowns, and nearly complete absence of roots of the maxillary teeth. d Rotational panoramic radiograph at the age of 23 after treatment showing four maxillary implants and a milled titanium superstructure

yngeal insufficiency. Thereupon, the hard and soft palate were reconstructed using a temporalis muscle flap. During that procedure, both coronoid processus were removed to improve mouth opening, and the remaining upper teeth and the maxillary osteosynthesis plates were removed. Because of several nose bleedings that occurred post surgery, a tracheotomy had to be placed for one and a half months.

Fifteen months after the reconstruction of the palate, four dental implants (Brånemark TiUnite regular platform, 
$13 \mathrm{~mm}$ ) were placed in the maxilla. Six months later, a maxillary overdenture on a milled titanium superstructure on four implants (Fig. 1d) was made. The patient was very satisfied with the final result and no problems occurred during the 3-year follow-up.

\section{Patient 2}

A 25-year-old female had been treated for a rhabdomyosarcoma in her nasal-tonsil region at the age of three. She was first treated with chemotherapy and subsequently with external beam radiotherapy (cumulative dose 59.4 Gy). This treatment resulted in a complete remission of the tumor. No recurrences or metastases were observed during a 22-year follow-up period. The late effects of the oncologic treatment were thin hair, development of cataract, impaired hearing at both sides, trismus, maxillary hypoplasia (Fig. 2a, b), hypodontia, microstomia, and malformation of teeth.

At the age of 20 years, the same combined surgical and prosthetic treatment plan was proposed to this patient as in patient 1 to correct the midface hypoplasia and to restore oral function. The patient, however, did not want to change her facial appearance, needing an alternative surgical treatment plan. This alternative plan consisted of a Le Fort I osteotomy with ventralization and down grafting of the maxilla to improve esthetics and jaw relationship for prosthetic rehabilitation instead of midface distraction with
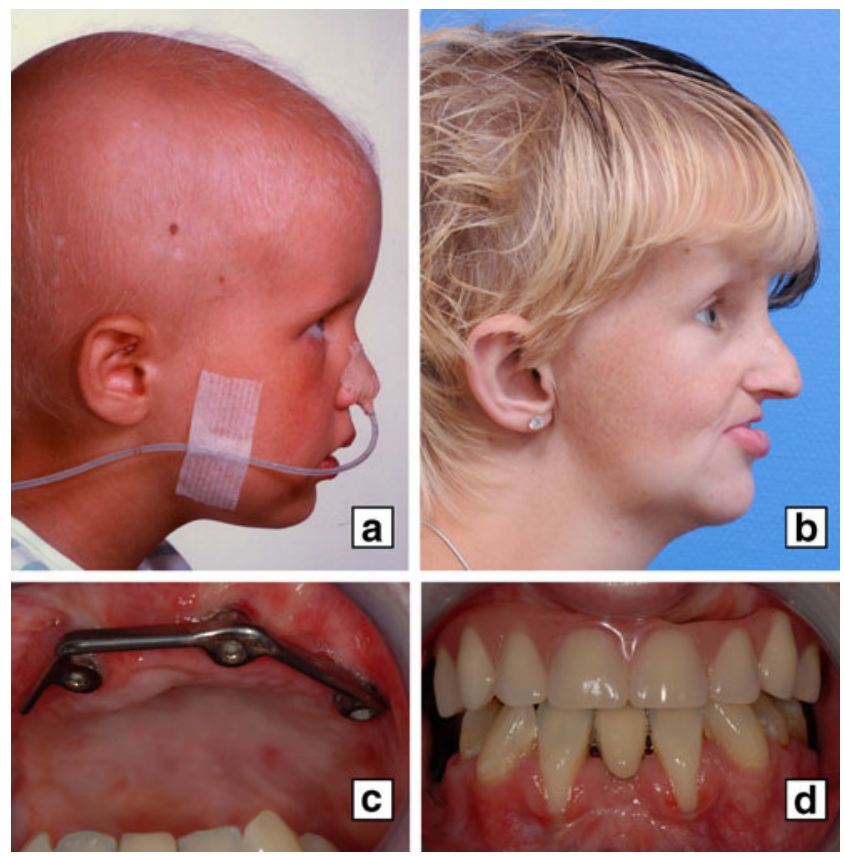

Fig. 2 a In profile image of the patient during treatment of the rhabdomyosarcoma at the age of 36 months. b In profile image of the patient at the age of 21 after oral rehabilitation, still showing midfacial hypoplasia. $\mathbf{c}$ The milled titanium superstructure on three implants. d The implant-based maxillary overdenture in situ the RED frame. The patient also refused this treatment and asked for dental rehabilitation only. Notwithstanding this suboptimal approach, it was assumed that the patient would greatly benefit from an implant-based prosthetic rehabilitation.

After 20 treatments with $\mathrm{HBO}$, the remaining maxillary teeth were removed. As the bone volume of the maxilla was insufficient for implant placement, 3 months after teeth extraction, bone from the iliac crest was used to lift the nasal floor and to broaden the maxilla to create bone volume allowing for a reliable placement of dental implants at the sites preferred by the prosthodontist. Three months later, four dental implants (Brånemark TiUnite regular platform, 10 and $13 \mathrm{~mm}$ ) were placed in the anterior maxilla with the help of a template, followed by ten treatments with HBO. After 5 months of osseointegration time, abutment connection was done and an impression was made. During this procedure, one implant appeared not to be osseointegrated and had to be removed. A milled titanium bar superstructure (Fig. 2c) and an overdenture (Fig. 2d) were made on the remaining three implants. The patient was very satisfied with her prosthesis and experienced no problems with her prosthetic rehabilitation during a two and a half-year follow-up.

\section{Discussion}

Radiotherapy in the head and neck region at a young age, either solely or combined with chemotherapy, can have severe side effects due to normal tissue damage as is obvious from both cases. When radiotherapy is delivered to the midface, facial growth retardation can cause esthetic and functional problems. Often, teeth are lost in spite of thorough oral hygiene care. Our first case shows that DO to correct midfacial hypoplasia can result in improved esthetics and can be beneficial to oral rehabilitation. Dental implant placement facilitates prosthetic treatment. The second case illustrates that if the patient refuses optimal treatment, a satisfactory result can occasionally also be obtained by implant-based prosthodontics.

The main advantage of applying DO is simultaneous soft tissue histogenesis that accompanies distraction of the bone [10]. DO in patients after tumor resection has been described for the mandible with varying results [11-14]. However, DO of the midface in irradiated patients is rarely reported [10]. The parameters of DO are empirically used in this case. Our patient showed satisfactory and stable bone formation and had improved facial esthetics.

Prosthetic rehabilitation of patients with a compromised oral situation can greatly benefit from placement of dental implants. In both patients described in this paper, a planning of a prosthetic rehabilitation with four implants was made in a multidisciplinary setting in cooperation with the 
surgeon and prosthodontist. When planning prosthetic rehabilitation, the most optimal position of the teeth should be the starting position. As both patients were able to maintain the teeth in the lower jaw, the dental configuration of the lower jaw was leading for the position of the teeth and the implants in the upper jaw.

A removable implant-based prosthesis was chosen in both of our patients because of more flexibility in positioning the teeth and fewer implants are needed compared to a fixed bridge. Also, in case of velopharyngeal insufficiency, a removable prosthesis allows for closing the defect, e.g., by combining the implant-retained removable prosthesis with an obturator. Good satisfaction levels can be achieved with removable implant-based prostheses as shown in our cases.

Both patients were treated with HBO before and after surgery. We used HBO treatment according to the Marx protocol, consisting of 20 dives before surgery and 10 dives after surgery [15]. In the literature, there is no consensus on the use of $\mathrm{HBO}$ to prevent osteoradionecrosis and to improve the success of implant treatment [16-19]. Most studies on these topics suggest a beneficial role for HBO, but these results need to be interpreted with caution. In the presented cases, we used HBO mainly to improve soft tissue healing after surgery [20].

\section{Conclusion}

Midfacial hypoplasia and loss of oral function resulting from the treatment of childhood maxillofacial rhabdomyosarcoma can be rehabilitated by a combination of orthognatic surgery, distraction osteogenesis, and implant-based prosthetics. A satisfying esthetical and functional result can be achieved with this treatment.

\section{Conflict of interest None.}

Open Access This article is distributed under the terms of the Creative Commons Attribution Noncommercial License which permits any noncommercial use, distribution, and reproduction in any medium, provided the original author(s) and source are credited.

\section{References}

1. Fyrmpas G, Wurm J, Athanassiadou F, Papageorgiou T, Beck JD, Iro H, Constantinidis J (2009) Management of paediatric sinonasal rhabdomyosarcoma. J Laryngol Otol 123:990-996

2. Paulino AC, Simon JH, Zhen W, Wen BC (2000) Long-term effects in children treated with radiotherapy for head and neck rhabdomyosarcoma. Int J Radiat Oncol Biol Phys 48:1489-1495

3. Healy JN, Borg MF (2010) Paediatric nasopharyngeal rhabdomyosarcoma: a case series and literature review. J Med Imaging Radiat Oncol 54:388-394
4. Kaste SC, Hopkins KP, Bowman LC (1995) Dental abnormalities in long-term survivors of head and neck rhabdomyosarcoma. Med Pediatr Oncol 25:96-101

5. Kaste SC, Goodman P, Leisenring W, Stovall M, Hayashi RJ, Yeazel M, Beiraghi S, Hudson MM, Sklar CA, Robison LL, Baker KS (2009) Impact of radiation and chemotherapy on risk of dental abnormalities: a report from the Childhood Cancer Survivor Study. Cancer 115:5817-5827

6. Schoen PJ, Raghoebar GM, Bouma J, Reintsema H, Burlage FR, Roodenburg JL, Vissink A (2008) Prosthodontic rehabilitation of oral function in head-neck cancer patients with dental implants placed simultaneously during ablative tumour surgery: an assessment of treatment outcomes and quality of life. Int $\mathrm{J}$ Oral Maxillofac Surg 37:8-16

7. Shetty K, Tuft H (2005) Dental management of the pediatric post radiation therapy - rhabdomyosarcoma patient: case reports and review of literature. Oral Oncol Extra 41:242-248

8. Estilo CL, Huryn JM, Kraus DH, Sklar CA, Wexler LH, Wolden SL, Zlotolow IM (2003) Effects of therapy on dentofacial development in long-term survivors of head and neck rhabdomyosarcoma: the Memorial Sloan-Kettering Cancer Center experience. J Pediatr Hematol Oncol 25:215-222

9. Carl W, Sako K, Schaaf NG (1974) Dental complications in the treatment of rhabdomyosarcoma of the oral cavity in children. Oral Surg Oral Med Oral Pathol 38:367-371

10. Vissink A, Burlage FR, Spijkervet FKL, Jansma J, Coppes RP (2003) Prevention and treatment of the consequences of head and neck radiotherapy. Crit Rev Oral Biol Med 14:213-225

11. Grover R, Murray D, Fialkov JA (2008) Distraction osteogenesis of radiation-induced orbitozygomatic hypoplasia. J Craniofac Surg 19:678-683

12. Gonzalez-Garcia R, Rodriguez-Campo FJ, Naval-Gias L, SastrePerez J, Diaz-Gonzalez FJ (2007) The effect of radiation in distraction osteogenesis for reconstruction of mandibular segmental defects. Br J Oral Maxillofac Surg 45:314-316

13. Holmes SB, Lloyd T, Coghlan KM, Newman L (2002) Distraction osteogenesis of the mandible in the previously irradiated patient. J Oral Maxillofac Surg 60:305-309

14. Raghoebar GM, Jansma J, Vissink A, Roodenburg JL (2005) Distraction osteogenesis in the irradiated mandible. A case report. J Craniomaxillofac Surg 33:246-250

15. Marx RE, Johnson RP, Kline SN (1985) Prevention of osteoradionecrosis: a randomized prospective clinical trial of hyperbaric oxygen versus penicillin. J Am Dent Assoc 111:49-54

16. Esposito M, Grusovin MG, Patel S, Worthington HV, Coulthard P (2008) Interventions for replacing missing teeth: hyperbaric oxygen therapy for irradiated patients who require dental implants. Cochrane Database Syst Rev (1):CD003603

17. Kashiwa K, Kobayashi S, Kimura H, Honda T, Nohara T, Fujiwara H, Hosoya Y, Arai Y (2007) Reconstruction of a severe maxillofacial deformity after tumorectomy and irradiation using distraction osteogenesis and LeFort I osteotomy before vascularized bone graft. J Craniofac Surg 18:1133-1137

18. Spiegelberg L, Djasim UM, van Neck HW, Wolvius EB, van der Wal KG (2010) Hyperbaric oxygen therapy in the management of radiation-induced injury in the head and neck region: a review of the literature. J Oral Maxillofac Surg 68:1732-1739

19. Schoen PJ, Raghoebar GM, Bouma J, Reintsema H, Vissink A, Sterk W, Roodenburg JL (2007) Rehabilitation of oral function in head and neck cancer patients after radiotherapy with implant-retained dentures: effects of hyperbaric oxygen therapy. Oral Oncol 43:379-388

20. Vissink A, Raghoebar GM, Roodenburg JL, Reintsema H, Spijkervet FK, Witjes MJ, Schoen PJ, Bouma J, Burlage FR, Langendijk JA (2009) Does hyperbaric oxygen therapy improve quality of life? Int J Oral Maxillofac Surg 38:99-100, author reply 100 